\title{
Micro Three-Dimensional Neuronal Cultures Generate Developing Cortex-Like Activity Patterns
}

\author{
Yixuan Ming ${ }^{1 *}$, Md Fayad Hasan', Svetlana Tatic-Lucic ${ }^{1,2}$ and Yevgeny Berdichevsky ${ }^{1,2 *}$ \\ ${ }^{1}$ Department of Electrical \& Computer Engineering, Lehigh University, Bethlehem, PA, United States, ${ }^{2}$ Department \\ of Bioengineering, Lehigh University, Bethlehem, PA, United States
}

\section{OPEN ACCESS}

Edited by:

Alexander Dityatev,

German Center

for Neurodegeneratives, Helmholtz Association of German Research

Centres (HZ), Germany

Reviewed by:

Dirk Schubert,

Radboud University Nijmegen,

Netherlands

Eleonora Vannini,

Institute of Neuroscience, Italian

National Research Council, Italy

*Correspondence:

Yixuan Ming

yim314@lehigh.edu

Yevgeny Berdichevsky

yeb211@lehigh.edu

Specialty section:

This article was submitted to

Neural Technology,

a section of the journal

Frontiers in Neuroscience

Received: 20 May 2020

Accepted: 09 September 2020

Published: 02 October 2020

Citation:

Ming Y, Hasan MF, Tatic-Lucic S and Berdichevsky Y (2020) Micro

Three-Dimensional Neuronal Cultures Generate Developing Cortex-Like

Activity Patterns.

Front. Neurosci. 14:563905. doi: 10.3389/fnins.2020.563905
Studies aimed at neurological drug discovery have been carried out both in vitro and in vivo. In vitro cell culture models have showed potential as drug testing platforms characterized by high throughput, low cost, good reproducibility and ease of handling and observation. However, in vitro neuronal culture models are facing challenges in replicating in vivo-like activity patterns. This work reports an in vitro culture technique that is capable of producing micro three-dimensional ( $\mu 3 \mathrm{D})$ cultures of only a few tens of neurons. The $\mu 3 \mathrm{D}$ cultures generated by this method were uniform in size and density of neurons. These $\mu 3 \mathrm{D}$ cultures had complex spontaneous synchronized neuronal activity patterns which were similar to those observed in the developing cortex and in much larger 3D cultures, but not in 2D cultures. Bursts could be reliably evoked by stimulation of single neurons. Synchronized bursts in $\mu 3 \mathrm{D}$ cultures were abolished by inhibitors of glutamate receptors, while inhibitors of $\mathrm{GABA}_{A}$ receptors had a more complex effect. This pharmacological profile is similar to bursts in neonatal cortex. Since large numbers of reproducible $\mu 3 \mathrm{D}$ cultures can be created and observed in parallel, this model of the developing cortex may find applications in high-throughput drug discovery experiments.

Keywords: 3D culture, neuron, calcium imaging, PDMS, microwell, burst, cortex, drug screening

\section{INTRODUCTION}

Developing cortex is characterized by bursts of spontaneous synchronized activity of populations of neurons, based on studies performed in pre-term infants and in neonatal rodents (Khazipov and Luhmann, 2006; Egorov and Draguhn, 2013; Luhmann et al., 2016; Arichi et al., 2017). Bursts in somatosensory and visual cortices may be driven by peripheral activity (Colonnese and Khazipov, 2012; Yang et al., 2016). However, cortex may also generate burst activity independently (Garaschuk et al., 2000; Lischalk et al., 2009; Namiki et al., 2013). This activity is important for neuronal maturation and formation of neural circuits, and may play a role in developmental neurological and psychiatric disorders (Kirischuk et al., 2017), some of which are characterized by altered neural activity (André et al., 2010). Experimental models of cortical bursts are therefore of high importance to elucidate burst mechanisms, to determine effects of bursts on neuronal circuits, and to develop therapies for disorders.

Development of therapies, in particular, requires that the model is characterized by high experimental throughput. Phenotypic screens are used in the drug development pipeline to identify promising lead in libraries consisting of hundreds or thousands of compounds (Parsons, 2019). The rapidity with which the effect of a compound on disease phenotype can be assessed determines 
the throughput of a screen. Current models of cortical bursts include direct in vivo measurements of cortical activity in rodent neonates, and in vitro measurements of activity in brain slices soon after dissection or after maintaining the slices in an incubator for several days or weeks (Table 1). These models are relatively labor-intensive, and are not scalable to high-throughput phenotypic screens. The challenge is then to develop a scalable model of cortical bursts that captures their salient features.

One possible candidate is the culture of dissociated neonatal rodent neurons. A single dissection generates millions of cortical neurons that can be maintained in a multi-well format, resulting in a high degree of parallelism suitable for high-throughput screens (Cooper et al., 2017). Dissociated neurons plated onto a treated substrate grow axons and dendrites and establish synaptic connections (Hasan and Berdichevsky, 2016). Resulting 2D networks are characterized by the presence of spontaneous activity that includes synchronized population bursts. However, bursts in $2 \mathrm{D}$ networks are qualitatively different than bursts in vivo or in slice models. When evaluated via $\mathrm{Ca}^{2+}$ imaging, $2 \mathrm{D}$ bursts appear as single-peak events with a decaying tail, while bursts in vivo or in slices are a mix of single-peak and multipeak events (Figure 1 and Table 1). When evaluated via electrical recordings, $2 \mathrm{D}$ bursts are synchronized events with short $(<1 \mathrm{~s})$ duration and typically a single-peak population spike (van Pelt et al., 2004; Wagenaar et al., 2006), while synchronized activity in the intact brain or slices includes multi-peak events lasting multiple seconds (Namiki et al., 2013), similar to immature human cortex (Vanhatalo et al., 2005). The presence of multiple population peaks in a burst may be due to the burst wave reflections from network edges, such as borders between different brain regions (Golshani et al., 2009; Namiki et al., 2013). The functional significance of multi-peak bursts may be that they result in significantly longer calcium elevations (Table 1) than single-peak bursts. Longer calcium transients ( $>10 \mathrm{~s}$ in cortical burst models vs. $\sim 1 \mathrm{~s}$ in $2 \mathrm{D}$ dissociated cultures) may initiate different intracellular signaling pathways linked to neuronal maturation and circuit formation.

We and others have previously shown that burst activity in three-dimensional (3D) cultures of dissociated neurons is qualitatively different from that in $2 \mathrm{D}$, and is characterized by the presence of prolonged, multi-peak, synchronized bursts (Wevers et al., 2016; Marom et al., 2017; Hasan et al., 2019). One approach to generate $3 \mathrm{D}$ cultures is to seed dissociated cells onto $3 \mathrm{D}$ scaffolds (Daud et al., 2012; Frega et al., 2014; Huang et al., 2014; Puschmann et al., 2014). However, limitation of this approach is that the spatial arrangement of cells in these $3 \mathrm{D}$ models is sparse, contradictory to in vivo models where cells are densely packed. An alternative approach is to seed dissociated cells into polydimethylsiloxane (PDMS) wells with diameter ranging from several hundred micrometers to millimeters (Park et al., 2015; Hasan et al., 2019). Three-dimensional cultures generated by this approach are relatively large in terms of physical size and the number of neurons, and are not well-suited for high throughput phenotypic screening. Large number of neurons may not be necessary for burst generation: synchronized domains as small as $100 \mu \mathrm{m}$ containing < 100 neurons have been reported in slices and in vivo (Corlew et al., 2004; Golshani et al., 2009).

Methods of forming 3D cultures smaller than $100 \mu \mathrm{m}$ have also been established. One of the well-known methods is the hanging drop method, in which dissociated cells are cultured in hanging droplets and form spheroids due to self-aggregation. One major limitation of this method is that it is labor-intensive and/or requires special-maintaining equipment because cells are cultured in droplets of around $20 \mu \mathrm{L}$ (Tung et al., 2011). Another approach of forming 3D cultures small than $100 \mu \mathrm{m}$ is to seed cells into microfabricated devices, the sizes of which are on micrometer scale. This approach has been widely used for various applications. For example, microwell arrays fabricated of or coated with non-adhesive materials, such as poly(ethylene glycol) (PEG) and agarose, have been used to study formation

TABLE 1 | Burst patterns and drug responses from previous in vitro and in vivo studies.

\begin{tabular}{|c|c|c|c|}
\hline References & Experimental details & Burst patterns & Drug response \\
\hline Garaschuk et al., 2000 & $\begin{array}{l}\mathrm{Ca}^{2+} \text { imaging of horizontal } \\
\text { slices from P1-2 rats. }\end{array}$ & $\begin{array}{l}\text { Interburst interval }(|\mathrm{B}|)=3.65 \mathrm{~s} \text {, up to } 15 \mathrm{~s} \text { burst } \\
\text { duration, single and multi-peak }\end{array}$ & $\begin{array}{l}\text { TTX blocked activity, Glutamatergic inhibitors } \\
\text { blocked activity, GABAergic inhibitors did not block }\end{array}$ \\
\hline Corlew et al., 2004 & $\begin{array}{l}\mathrm{Ca}^{2+} \text { imaging of horizontal } \\
\text { slices from } \mathrm{PO} \text { rats }\end{array}$ & $\begin{array}{l}\mathrm{IBI}=1.1 \mathrm{~min}, 5-15 \mathrm{~s} \text { burst duration, single and } \\
\text { multi-peak }\end{array}$ & TTX blocked activity \\
\hline Allene et al., 2008 & $\begin{array}{l}\mathrm{Ca}^{2+} \text { imaging of horizontal } \\
\text { slices from E20-P9 rats }\end{array}$ & $\begin{array}{l}\text { Data from } \mathrm{P} 0-3 \text { rats: } \mathrm{IBI}=0.41 \mathrm{~min} \text {, up to } 10 \mathrm{~s} \\
\text { burst duration, single and multi-peak }\end{array}$ & $\begin{array}{l}\text { Glutamatergic inhibitors blocked activity, } \\
\text { GABAergic inhibitors did not block }\end{array}$ \\
\hline Namiki et al., 2013 & Horizontal slices from P1-6 rats & $\begin{array}{l}\mathrm{IBI}=0.42 \text { min, up to } 10 \mathrm{~s} \text { burst duration, single and } \\
\text { multi-peak }\end{array}$ & $\begin{array}{l}\text { TTX blocked activity, Glutamatergic inhibitors } \\
\text { blocked activity, GABAergic inhibitors did not block }\end{array}$ \\
\hline Adelsberger et al., 2005 & $\begin{array}{l}\text { Optical fiber recording from } \\
\text { P3-4 mouse }\end{array}$ & $\begin{array}{l}\mathrm{IBI}=0.44 \text { min (from examples), } 3.8 \pm 0.1 \mathrm{~s} \text { burst } \\
\text { duration (longest example is } 16 \mathrm{~s} \text { ), single and } \\
\text { multi-peak }\end{array}$ & N.A. \\
\hline Conhaim et al., 2011 & $\begin{array}{l}\text { Organotypic cultures from E17 } \\
\text { mice, evaluated for } 14 \text { days } \\
\text { in vitro }\end{array}$ & $\begin{array}{l}\text { IBls vary widely. Shortest: } 0.25 \text { min. Up to } 9.3 \mathrm{~s} \\
\text { burst duration, single and multi-peak }\end{array}$ & $\begin{array}{l}\text { Glutamatergic inhibitors inhibited, but most effective } \\
\text { (complete block) after DIV 9, GABAergic inhibitors } \\
\text { blocked until DIV 8, then stimulated }\end{array}$ \\
\hline Golshani et al., 2009 & $\begin{array}{l}\mathrm{Ca}^{2+} \text { imaging of mice from } \mathrm{P} 4 \\
\text { to adulthood }\end{array}$ & $\begin{array}{l}\text { In neonates, }|\mathrm{B}|=0.63 \mathrm{~min} \text { (from examples), up to } \\
10.6 \mathrm{~s} \text { burst duration, single and multi-peak }\end{array}$ & N.A. \\
\hline Sheroziya et al., 2009 & $\begin{array}{l}\text { Electrical recordings in } \\
\text { horizontal slices of } \mathrm{P} 1-13 \text { rats }\end{array}$ & $\begin{array}{l}|\mathrm{B}|=0.28 \mathrm{~min} \text { for } \mathrm{P} 5-7,3.5 \pm 1.2 \mathrm{~s} \text { burst duration } \\
\text { at } \mathrm{P} 5-7 ; 4.8 \pm 2.5 \mathrm{~s} \text { at } \mathrm{P} 11-13 \text {, single and } \\
\text { multi-peak }\end{array}$ & $\begin{array}{l}\text { TTX blocked activity, Glutamatergic inhibitors } \\
\text { blocked activity, GABAergic inhibitors elicited } \\
\text { discharges at P5-7 }\end{array}$ \\
\hline
\end{tabular}




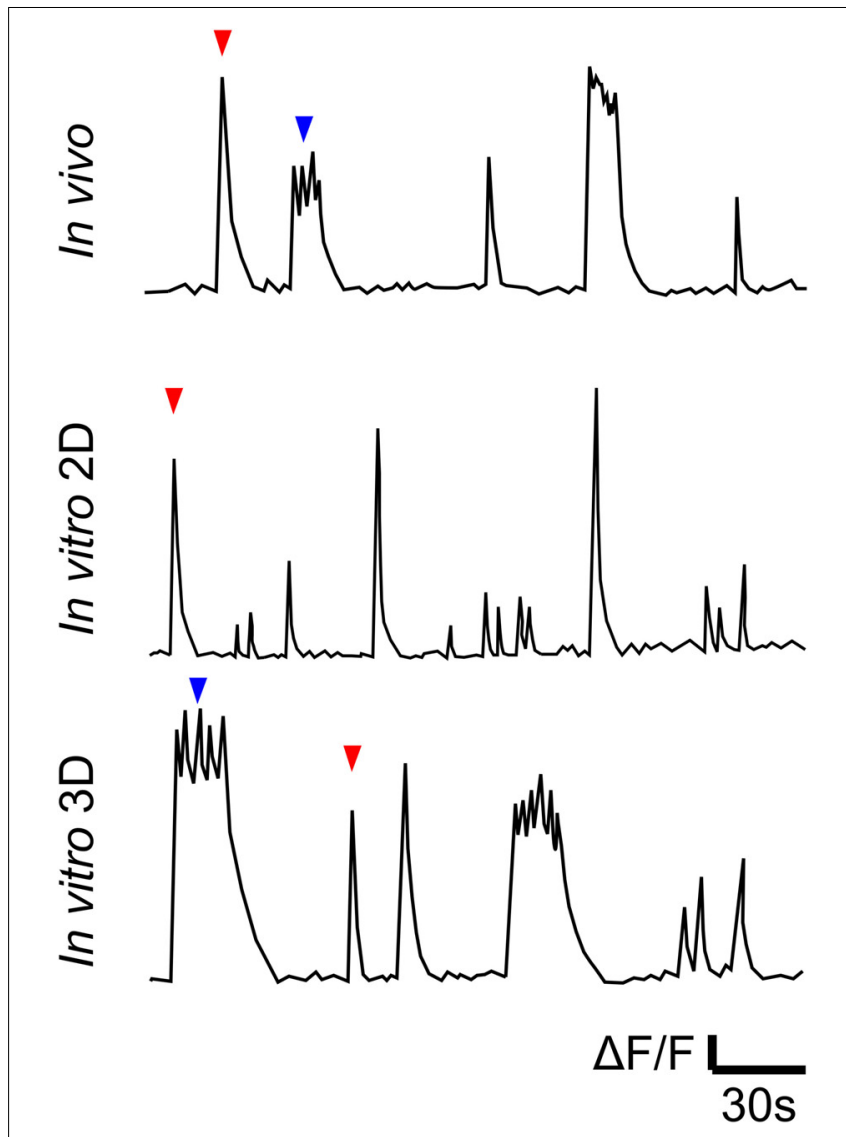

FIGURE 1 | Cartoon illustration of $\mathrm{Ca}^{2+}$ traces of in vivo (representative of data in references in Table 1), in vitro 2D and in vitro 3D models (Hasan et al., 2019). Traces illustrate qualitative differences between spontaneous bursts in 2D cultures, on the one hand, and in vivo and in 3D cultures, on the other. Red and blue arrows indicate single- and multi-peak events, respectively.

of homogeneously sized embryonic bodies (EBs) from murine embryonic stem cells (mESCs) (Karp et al., 2007; Moeller et al., 2008; Sakai et al., 2010; Pasturel et al., 2019), and effect of size on their differentiation (Karp et al., 2007; Jeong et al., 2012). Formation and differentiation of EBs have also been investigated with microwells attached to adhesive substrates (Mohr et al., 2006; Park et al., 2007). Neurospheres formed from murine neural progenitor/stem cells (mNPCs) in PEG microwells have been studied for self-renewal ability and multipotency (Cordey et al., 2008). Microwells and microfluidic devices have also been utilized to form in vitro 3D disease models from pancreatic islets cells (Ichihara et al., 2016), hepatocytes (Sakai et al., 2010), human embryonic kidney (HEK) cells (Pasturel et al., 2019), human hepatoblastoma cells (HepG2) (Sakai et al., 2010; Selimović et al., 2011; Ong et al., 2017), and cortical neurons (Choi et al., 2013). Networks formed with neurospheres that are around $100 \mu \mathrm{m}$ in diameter have been studied (Kato-Negishi et al., 2010), where neurospheres were harvested from PDMS microwells and cultured on polyethyleneimine (PEI)-coated glass plates. Neighboring neurospheres formed random connections and synchronized spontaneous activities were observed. Although 3D cultures of cortical neurons that are less than $100 \mu \mathrm{m}$ in size have been reported (Kato-Negishi et al., 2010; Choi et al., 2013; Pasturel et al., 2019), little has been done to evaluate the activity patterns. Also, these 3D cultures were connected by sprouting axons into a single network, limiting the throughput of this platform for drug discovery.

In this work, we report a novel method of creating micro$3 \mathrm{D}(\mu 3 \mathrm{D})$ cultures of dissociated neurons with dimensions below $100 \mu \mathrm{m}$. These $\mu 3 \mathrm{D}$ cultures are closely spaced for simultaneous optical evaluation, but are not connected with axons and represent separate, independent networks. We then evaluate activity in $\mu 3 \mathrm{D}$ cultures to determine if they model developmental cortical bursting. Our evaluation criteria included burst waveform (single vs. multi-peak), burst duration, and dependence on voltage-gated sodium channels and glutamatergic or GABAergic neurotransmission, as reported for developmental cortical bursts (Table 1).

\section{MATERIALS AND METHODS}

\section{SU-8 Master and PDMS Microstructure Fabrication}

First, an 80- $\mu$ m-thick layer of SU-8 2050 (Microchem Corp.) was spun onto a 3 -inch silicon wafer at 4,000 revolutions per minute (RPM) for $1 \mathrm{~min}$. Then, the wafer was baked on a hotplate at $65^{\circ} \mathrm{C}$ for $3 \mathrm{~min}$ and $95^{\circ} \mathrm{C}$ for $6 \mathrm{~min}$. After cooling down to room temperature, the wafer was exposed to ultraviolet (UV) light through a mask for a total exposure energy of $160 \mathrm{~mJ} \mathrm{~cm}{ }^{-2}$. Next, the wafer was baked on a hotplate at $65^{\circ} \mathrm{C}$ for $1 \mathrm{~min}$, followed by baking on $95^{\circ} \mathrm{C}$ for $6 \mathrm{~min}$, and then cooled down to room temperature. After development in SU-8 developer (Microchem Corp.) for $5 \mathrm{~min}$, only SU- 8 patterns defined by the mask stayed on the wafer. The SU-8 patterns were rinsed with Isopropyl Alcohol (IPA) and dried with nitrogen. Finally, the SU-8 master was hard baked at $120^{\circ} \mathrm{C}$ overnight, followed by silanization for $16 \mathrm{~h}$ at room temperature in a vacuum desiccator filled with trichloro $(1 \mathrm{H}, 1 \mathrm{H}, 2 \mathrm{H}, 2 \mathrm{H}$-perfluorooctyl)silane (Sigma-Aldrich).

Polydimethylsiloxane (PDMS) base was mixed with curing agent (Dow Corning Corp.) at 10:1 ratio and left in a vacuum desiccator for $15 \mathrm{~min}$ to remove bubbles. The mixture was then spun onto the SU- 8 master at 1000 RPM for 3 min and cured at $75^{\circ} \mathrm{C}$ overnight, leading to an $80 \mu \mathrm{m}$ thick PDMS layer. PDMS microstructures were carefully peeled off from the master, cut into desired size and sterilized in ethanol. After drying, a piece of PDMS microstructure was carefully lowered onto a coverslip coated with poly-D-lysine (PDL, Sigma-Aldrich) (Figure 2A). A second layer of PDMS microstructure, which is slightly smaller (for the ease of handling), was aligned on top of the first one under a stereo microscope (Figure 2B). The second layer served as a cellular mask and was removed after cell seeding, which prevents cells from attaching to undesired locations. Finally, the structure was placed into a 35-mm-diameter Petri dish, immersed under cell culture media [97.5\% Neurobasal-A media (Invitrogen), 2\% B27, $1 \mathrm{mM}$ glutaMAX (Gibco) and $30 \mu \mathrm{g} / \mathrm{mL}$ gentamicin (Gibco)] and incubated at $37^{\circ} \mathrm{C}$ overnight to reduce cytotoxicity. 


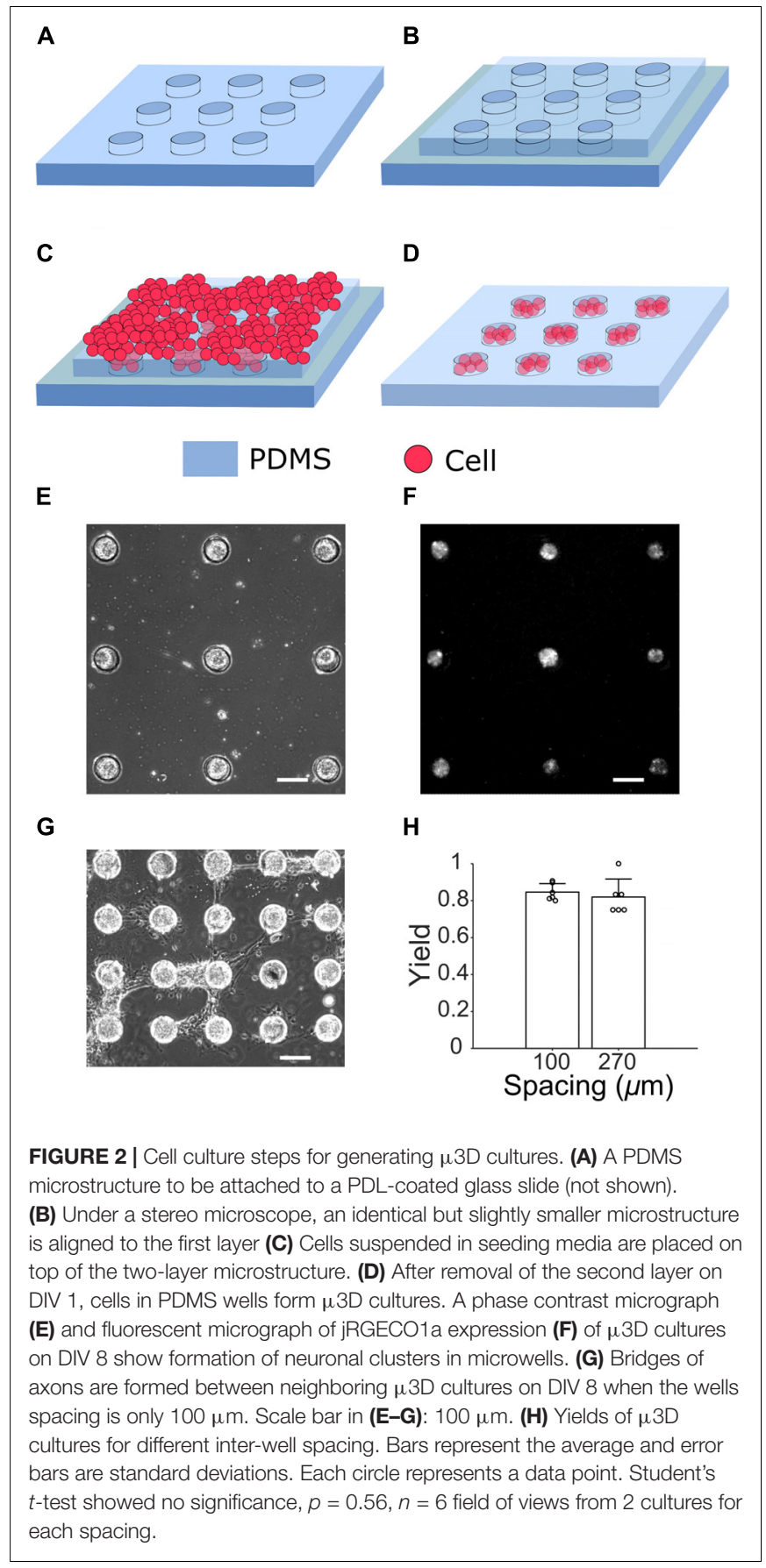

\section{Cell Dissociation and Culture}

Cortices were obtained from postnatal day 0-1 (p0-1) SpragueDawley rat pups (Charles River Laboratories) per the protocol described in Brewer et al. (1993). All animal use protocols were approved by the Institution Animal Care and Use Committee (IACUC) at Lehigh University and were conducted in accordance with the United States Public Health Service Policy on Humane Care and Use of Laboratory Animals. Cortices were then cut and dissociated with papain (Worthington Biochemical Corp.) in Hanks' balanced salt solution (HBSS, Gibco) for $20 \mathrm{~min}$.
The dissociated cortices were washed with fresh media three times. Cells were obtained from the dissociated cortices after trituration and centrifugation. Next, cells were resuspended with cell seeding media [Neurobasal-A, $0.5 \mathrm{mM}$ GlutaMAX, $30 \mu \mathrm{g} / \mathrm{mL}$ gentamicin and 10\% Fetal Bovine Serum (FBS, Gibco)] at desired concentration. Method for generating $1300 \mu \mathrm{m} 3 \mathrm{D}$ cultures was published elsewhere (Hasan et al., 2019). For 2D cultures, cells were seeded onto a PDL-coated cover slip at $0.5 \mathrm{M} / \mathrm{mL}$. For $\mu 3 \mathrm{D}$ cultures, a total number of $0.508 \mathrm{M}$ cells and $20 \mu \mathrm{L}$ of cell seeding media were added inside the PDMS device described in the previous section (Figure 2C). After $15 \mathrm{~min}$, another $2 \mathrm{~mL}$ of cell seeding media was added to the dish. Cells were allowed to settle down for $45 \mathrm{~min}$ and then cell seeding media was replaced with $2 \mathrm{~mL}$ fresh culture media (see section "SU-8 Master and PDMS Microstructure Fabrication" for the recipe). One day after cell plating, cellular mask was carefully removed (Figure 2D). Cell culture was incubated at $37^{\circ} \mathrm{C}$ with $5 \% \mathrm{CO}_{2}$, and half of the culture media was replaced twice per week.

\section{Immunocytochemistry (ICC) and Cell Counting}

Cell cultures were fixed at room temperature with $4 \%$ paraformaldehyde (PFA, Electron Microscopy Science) in phosphate buffered saline (PBS, Sigma-Aldrich) for $1 \mathrm{~h}$, and then permeabilized in $0.3 \%$ Triton X-100 in PBS for 15 min. Next, cultures were blocked with $10 \%$ goat serum in $0.05 \%$ Triton $\mathrm{X}-100$ in PBS. Primary antibodies anti-MAP2 (1:2000 dilution, BioLegend), SMI312 (anti-Neurofilament Marker antibody, 1:500 dilution, BioLegend), anti-GFAP (Anti-Glial Fibrillary Acidic Protein Antibody, 1:500 dilution, Sigma-Aldrich) or AntiGAD67 (1:500 dilution, Sigma-Aldrich) were added to the culture which was then kept at $4^{\circ} \mathrm{C}$ for 3 days. After wash, secondary antibodies were added accordingly. The culture was kept at $4^{\circ} \mathrm{C}$ for another 3 days. Finally, DAPI (Invitrogen) was added after wash. Cultures were then washed, mounted and imaged on a confocal microscope (Zeiss LSM 510 META, Germany). Three dimensional reconstruction of cell cultures was performed in ZEN lite (Zeiss).

Cell counting was done manually by analyzing confocal images in Image) (Schneider et al., 2012). DAPI-positive objects were counted as cells with tiny and bright ones excluded. Objects that were MAP2-positive $\left(\mathrm{MAP2}^{+}\right)$or jRGECO1a-expressing and co-localized with a cell count, were marked as neurons. Curve fitting of number of cells to seeding concentration and drug response of phenytoin were done in MATLAB using the curve fitting toolbox. GFAP-positive (GFAP+) cells and $($ GAD67+) cells were counted as astrocytes and GABAergic neurons, respectively.

\section{Whole Cell Recording}

Whole cell recordings were performed on DIV 18-20. Upright microscope (Olympus BX51WI, Olympus Optics, Japan) equipped with infrared-differential interference contrast optics was used to locate neurons in $\mu 3 \mathrm{D}$ cultures. The recordings were conducted at $32-34^{\circ} \mathrm{C}$, and the resistance of the recording 
pipette (1.5 mm borosilicate glass, Sutter Instruments) was 4 to $7 \mathrm{M} \Omega$. The cultures were held in a perfusion chamber with constant flow of recording solution composed of: $120 \mathrm{mM} \mathrm{NaCl}$, $3.5 \mathrm{mM} \mathrm{KCl}, 1.3 \mathrm{mM} \mathrm{CaCl} 2,0.9 \mathrm{mM} \mathrm{MgCl}_{2}, 25 \mathrm{mM} \mathrm{NaHCO}_{3}$, $1.23 \mathrm{mM} \mathrm{NaH}_{2} \mathrm{PO}_{4}$, and $10 \mathrm{mM}$ glucose, bubbled with $5 \%$ $\mathrm{CO}_{2} 21 \% \mathrm{O}_{2}$, and balanced with $\mathrm{N}_{2}$. In some experiments, $3 \mathrm{mM}$ kynurenic acid (KYNA) was added to recording solution to prevent network bursts. The recording pipette was loaded with intracellular solution containing $130 \mathrm{mM} \mathrm{K}$-gluconate, $5 \mathrm{mM} \mathrm{KCl}, 4 \mathrm{mM}$ ATP-Mg, $0.3 \mathrm{mM}$ GTP, $10 \mathrm{mM}$ HEPES, and $10 \mathrm{mM}$ phosphocreatine ( $\mathrm{pH} 7.2$, adjusted with $\mathrm{KOH}$ ). Up to 2 cells were patched from each culture. Current-voltage curves were recorded in current clamp mode using MultiClamp 700B (Molecular Devices) and acquired at sampling frequency of $20 \mathrm{kHz}$ through DigiData 1550B and pCLAMP 10 software (Molecular Devices). The data was analyzed in pCLAMP 10, without correction for liquid junction potential.

\section{Optical Recording}

To record neural activity optically, cells were infected on day in vitro (DIV) 1 with adeno-associated virus (AAV) to express red genetically encoded calcium indicator for optical recording (jRGECO1a) under Syn promoter [AAV9, pAAV.Syn.NES-jRGECO1a.WPRE.SV40, which was a gift from The Genetically Encoded Neuronal Indicator and Effector Project (GENIE) \& Douglas Kim (Addgene viral prep \# 100854-AAV9'; RRID:Addgene_100854)]. Dynamic fluorescence changes of jRGECO1a caused by neuronal activities could be observed from

${ }^{1}$ http://n2t.net/addgene: 100854 around DIV 8. To record the fluorescence changes, the dish containing infected cell culture was placed into a mini incubator chamber (Bioscience Tools). The chamber was maintained at $37^{\circ} \mathrm{C}$ and supplied with humidified gas mixture $\left(5 \% \mathrm{CO}_{2}, 21 \% \mathrm{O}_{2}\right.$ and balanced $\mathrm{N}_{2}$, Airgas). Fluorescence intensity was recorded for $12 \mathrm{~min}$ with an inverted microscope (Olympus).

\section{Burst Detection and Analysis}

To extract fluorescence intensities from optical recordings, regions of interest were drawn around the whole $\mu 3 \mathrm{D}$ culture or single cells in 2D culture. Mean gray value $F$ of each $3 \mathrm{D}$ culture and each cell in $2 \mathrm{D}$ cultures was measured using ImageJ (Schneider et al., 2012). Baseline $F_{0}$ of each recording was obtained using MATLAB and the algorithm described in Eilers and Boelens (2005). Fluorescence change, $\Delta F / F$, was calculated by:

$$
\Delta \mathrm{F} / \mathrm{F}=\left(\mathrm{F}-\mathrm{F}_{0}\right) / \mathrm{F}_{0}
$$

A threshold of mean plus two times standard deviation of the baseline was set to detect activities. When the fluorescence surpassed the threshold, it was considered as the start of an activity and the activity was considered to end when the fluorescence dropped below the threshold.

For $\mu 3 \mathrm{D}$ cultures and large 3D cultures, fluorescence changes were interpreted as network-wide synchronized neuronal activities or population bursts (referred as bursts). For 2D cultures, activities of single cells were detectable. Only activities involving more than $50 \%$ of the total neurons within the network were considered as population bursts (or bursts) and used for data analysis.
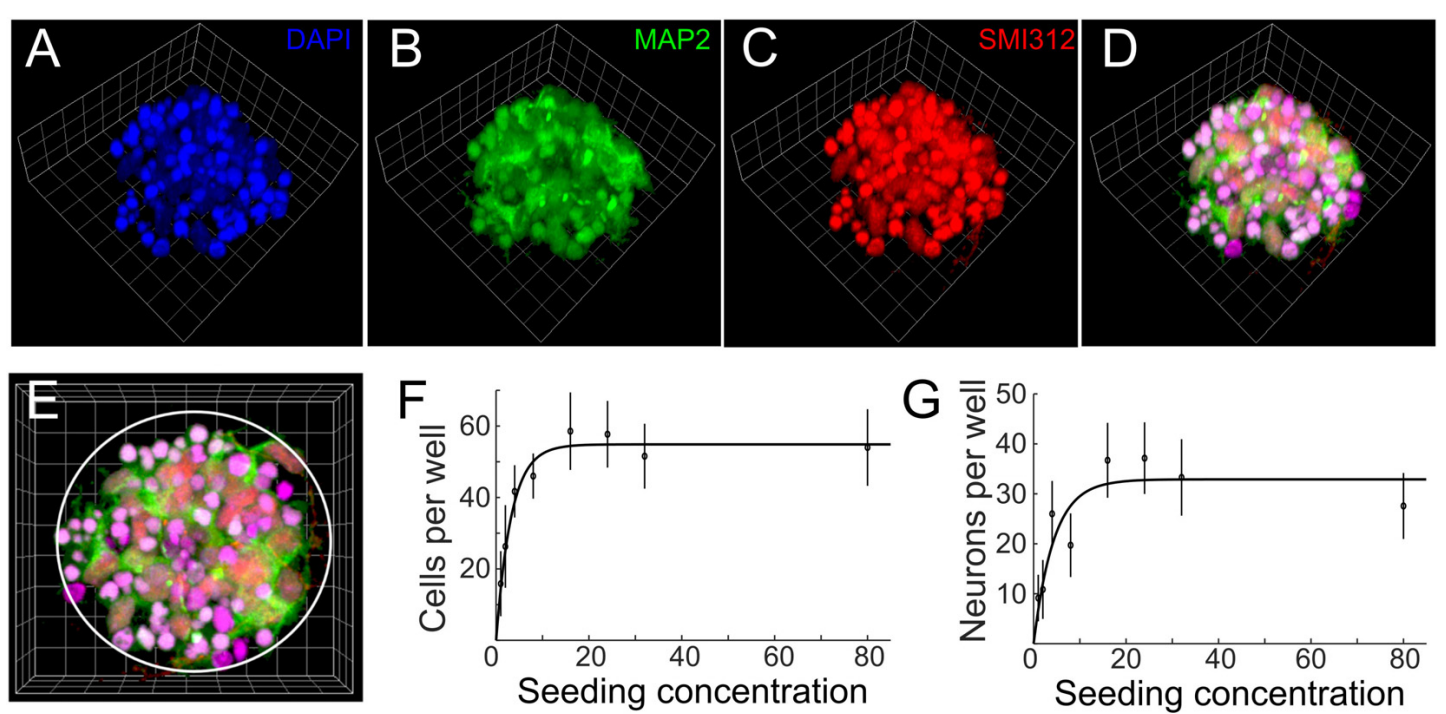

$\left(10^{\wedge} 6\right.$ cells $\left./ \mathrm{mL}\right)$

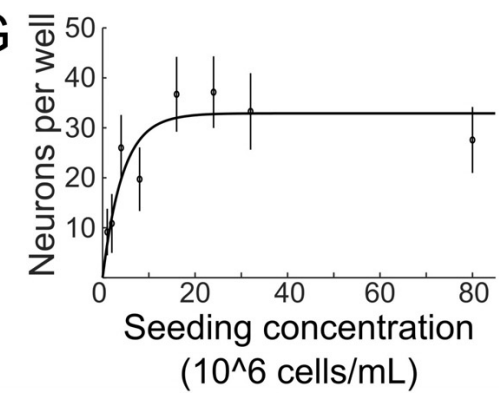

FIGURE 3 | (A-D) 3D reconstruction of a cell cluster in a PDMS well. Side length of the reference squares is $7.5 \mu \mathrm{m}$. Cells were fixed on DIV 3 and stained with (A) DAPI (blue, nuclei), (B) anti-MAP2 (green, soma and dendrites of neurons) and (C) SMI312 (red, soma and axons of neurons). Colocalization of green, red and blue is displayed as magenta in panel (D). (E) Top view of the $\mu 3 \mathrm{D}$ culture. White circle denotes boundary of the PDMS well. Average number of cells (F) and neurons (G) per well at different seeding concentrations. Error bars are standard deviations. $n=7 \mu 3 \mathrm{D}$ cultures for each seeding concentration. Solid lines are curves fitted to exponential functions using least absolute residuals (LAR) method. $r^{2}=0.96, p=1.61 \times 10^{-5}$ for $(\mathbf{F})$ and $r^{2}=0.77, p=4.1 \times 10^{-3}$ for $(\mathbf{G})$. 

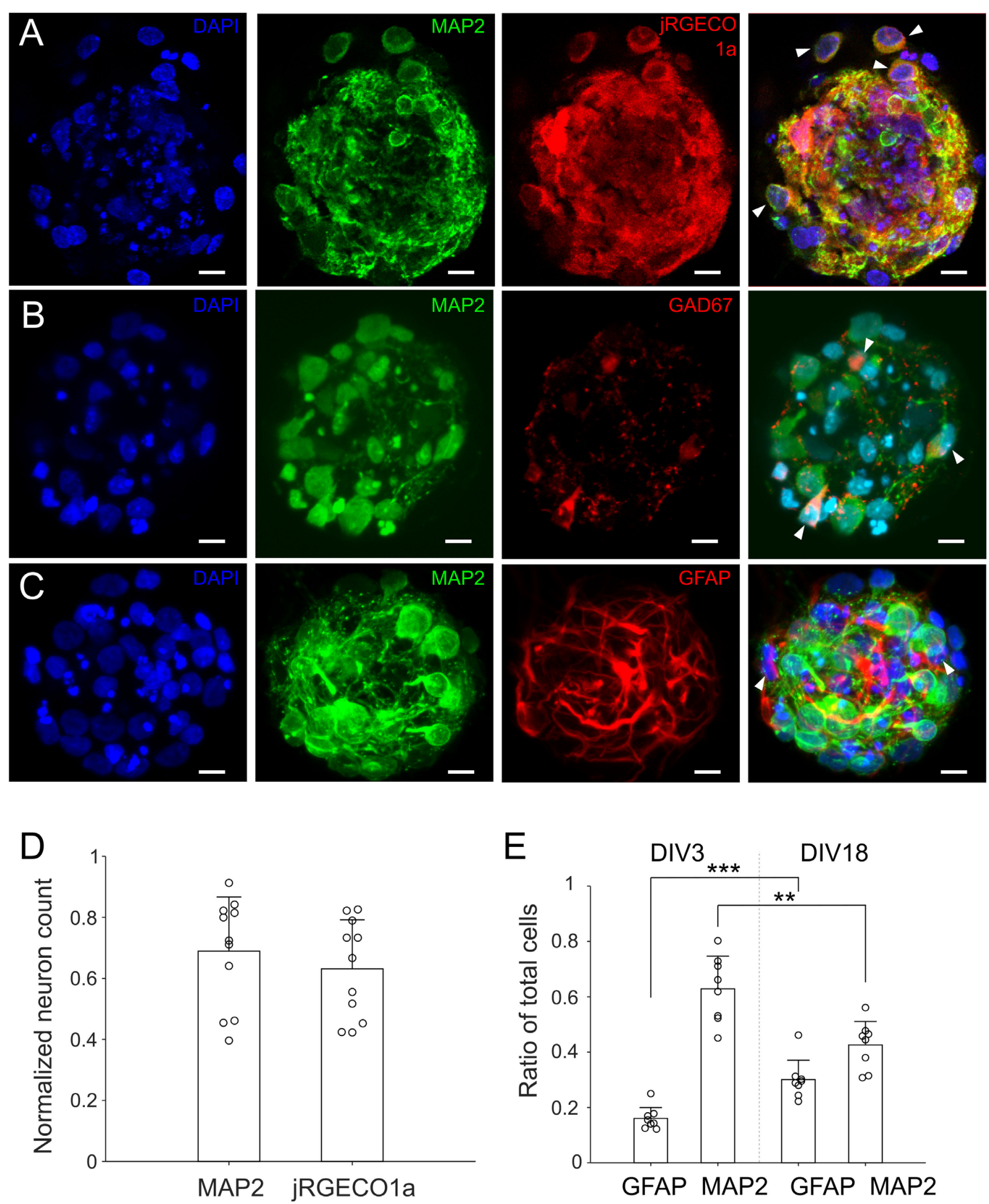

FIGURE 4 | Neurons and astrocytes in $\mu 3 D$ cultures. (A) Cultures were infected by AAV9 with jRGECO1a on DIV 1, fixed on DIV 14 and stained with DAPI (blue, nuclei) and anti-MAP2 (green, soma and dendrites of neurons). On right is the merge of DAPI, anti-MAP2 and jRGECO1a fluorescence, with white arrows indicating MAP2 $^{+}$cells (neurons) expressing jRGECO1a. (B) Cultures were fixed on DIV 18 and stained with DAPI, anti-MAP2 and anti-GAD67 (GABAergic neuron marker). White arrows in the merged image indicate GABAergic neurons (MAP2 ${ }^{+} /{\text {GAD } 67^{+}}^{\text {). (C) }}$ Cultures were fixed on DIV 18 and stained with DAPI, anti-MAP2 and anti-GFAP (astrocyte marker). White arrows in the merged figure indicate examples of astrocytes (MAP2-/GFAP ${ }^{+}$). (A-C) Are $\mathbf{Z}^{-}$-stacks of 5-10 $\mu \mathrm{m}$ thick. (D) Neuron counts based on anti-MAP2 and jRGECO1a expression, which were normalized by corresponding cell counts (DAPI). Bars represent the averages and error bars the standard deviations. Each circle represents a $\mu 3 \mathrm{D}$ culture. Student's t-test showed no significance, $p=0.43, n=11$. Scale bar: $15 \mu \mathrm{m}$. (E) Ratio of astrocytes and neurons on DIV 3 and 18. Student's $t$-tests were used to evaluate statistical significance. ${ }^{* \star} p<0.01$ and ${ }^{\star \star \star} p<0.001, n=8$ cultures for each DIV. 


\section{Pharmacological Experiments}

Cultures were recorded in regular culture media (vehicle) first. Next, they were incubated for $30 \mathrm{~min}$ in culture media containing kynurenic acid (KYNA, $3 \mathrm{mM}$, Sigma-Aldrich) or bicuculline $(10 \mu \mathrm{M}$, Tocris) and recorded in the same media. Next, cell cultures were washed three times with fresh regular media and incubated for $2 \mathrm{~h}$ with regular culture media before the final (wash-out) recording.

For the APV [D-(-)-2-Amino-5-phosphonopentanoic acid, Tocris] and NBQX (2,3-Dioxo-6-nitro-1,2,3,4tetrahydrobenzo[f]quinoxaline-7-sulfonamide, Tocris) experiment, cultures were recorded in regular culture media (vehicle) first. Then, they were incubated for $30 \mathrm{~min}$ with culture media containing APV $(50 \mu \mathrm{M})$ and recorded in the same media. Next, cultures were incubated for another $30 \mathrm{~min}$ with culture media containing APV $(50 \mu \mathrm{M})$ and NBQX $(10 \mu \mathrm{M})$ and recorded. Finally, cultures were washed three times with fresh culture media, incubated for $2 \mathrm{~h}$ with regular culture media and recorded for the wash-out recording.

To measure the response of $\mu 3 \mathrm{D}$ cultures to phenytoin (10, $20,50,100$, and $150 \mu \mathrm{M}$, Sigma-Aldrich), cultures were first recorded in regular culture media. Then, cultures were incubated for $1 \mathrm{~h}$ in culture media containing the lowest concentration of phenytoin. After the incubation, they were recorded. Next, culture medium was changed to medium with next higher concentration of phenytoin and cultures were recorded after another $1 \mathrm{~h}$ of incubation. The process was repeated until the highest concentration of phenytoin was tested. Finally, cultures were washed three times with fresh media and recorded for the final (wash-out) recording. Data were collected for analysis from cultures that were active for both vehicle and washout recordings.

\section{RESULTS}

When microwells were distributed sparsely $(270 \mu \mathrm{m}$ spacing in Figure 2E), they enabled good confinement for cells and neurites. However, when microwells were too close to each other, neurites of cell clusters were able to climb over microwell walls and formed bridges which connected neighboring cell clusters (100 $\mu \mathrm{m}$ spacing in Figure 2G). The bridges of neurites were observed starting from DIV 6 when spacing between microwells was $100 \mu \mathrm{m}$. The yield (\% of microwells with $\mu 3 \mathrm{D}$ neuron cluster) was calculated based on phase contrast pictures taken on DIV 6 (Figure $\mathbf{2 H}$ ). There is no significant difference between average yields of $100-\mu \mathrm{m}$-spacing $(0.846)$ and $270-\mu \mathrm{m}$-spacing (0.819) microwells. High yield is evidence that this method generates $\mu 3 \mathrm{D}$ cultures reliably and robustly.

To characterize the morphology and quantify number of cells and neurons of $\mu 3 \mathrm{D}$ cultures, cell cultures were fixed on DIV 3, co-stained with anti-MAP2 for soma and dendrites of neurons, SMI312 for axons of neurons and DAPI for nuclei (Figures 3A-E), and imaged with a confocal microscope. The seeding concentration for the $\mu 3 \mathrm{D}$ culture shown in Figures $3 \mathrm{~A}-\mathrm{E}$ is $2.4 \times 10^{\wedge} 7$ cells $/ \mathrm{mL}$. Cell counting was done manually in ImageJ. Since small and bright DAPI positive objects indicate shrunk nuclei of unhealthy cells (Casiano et al., 1998), only cells with morphologically normal nuclei were included in the cell count. $\mathrm{MAP}^{+}$objects were counted as neurons if they contained a morphologically normal nucleus. Number of cells and neurons per well and the corresponding seeding concentration are shown in Figures 3F,G, in which circles represent data points and lines are curves fitted to exponential functions. Number of cells per well increased with the seeding concentration and reached the maximum of 58.6 cells per well at seeding concentration of $1.6 \times 10^{\wedge} 7$ cells $/ \mathrm{mL}$. Number of cells did not change significantly when seeding concentration exceeded $1.6 \times 10^{\wedge} 7$ cells $/ \mathrm{mL}$, likely because the maximum capacity of the PDMS microwell was reached. Similarly, number of neurons per well increased with the seeding concentration and reached the maximum of around 37.1 neurons per well at seeding concentration of $1.6 \times 10^{\wedge} 7 \mathrm{cells} / \mathrm{mL}$ (Figure 3G). Aiming at efficiently generating $\mu 3 \mathrm{D}$ cultures containing maximum number of neurons, the seeding concentration was then set to $1.6-2.4 \times 10^{\wedge} 7$ cells $/ \mathrm{mL}$ for PDMS microwells of $70 \mu \mathrm{m}$ diameter and $80 \mu \mathrm{m}$ height. At this seeding concentration, the ratio of standard deviation to average number of neurons in the culture is 0.19 , which speaks to the reproducibility of this culture method. If the volume of PDMS wells increases, it is expected that the size of $\mu 3 \mathrm{D}$ cultures and average number of cells and neurons would also increase. Thus, different sizes of $\mu 3 \mathrm{D}$ cultures may be generated by varying the volume of the PDMS microwells and the corresponding seeding concentration.

For optically recording neuronal activities, cultures were infected by AAV9 with jRGECO1a on DIV 1 after removal of the cellular mask. Cultures expressing jRGECO1a were stained with anti-MAP2 on DIV 14 (Figure 4A). Neuron counting based on anti-MAP2 staining and jRGECO1a expression yielded
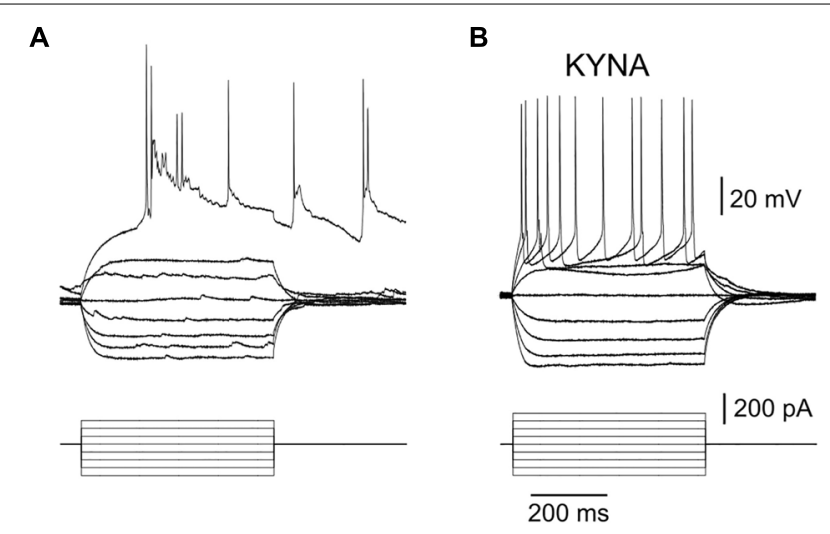

FIGURE 5 | Burst triggered by a single action potential. (A) Bottom: injected current pulses (current clamp mode, base injected current is zero), top: recorded voltage shows that 1st evoked AP triggered a burst when culture was in a regular recording solution. Resting membrane potential (baseline of the voltage curves) for this neuron was $-65.1 \mathrm{mV}$. Results are representative of 8 IV curves obtained in 4 neurons. (B) No bursts were triggered when $3 \mathrm{mM}$ KYNA was added to the recording solution (resting membrane potential (baseline of voltage curves) was $-64.8 \mathrm{mV}$ in this recording), results are representative of $n=9$ neurons. 
similar results (Figure 4D). The average percentage of antiMAP2 positive neurons that were also expressing jRGECO1a was $93 \% \pm 14.8 \%$, indicating that expression of jRGECO1a was of high efficiency, and that activity of nearly all neurons in culture contributed to the recorded activities. Ratio of GABAergic to glutamatergic neurons was $0.18 \pm 0.05$ ( $n=20$ cultures, Figure 4B), similar to the ratio reported in earlier, larger in vitro 3D culture model (Frega et al., 2014). Astrocytes and their
A

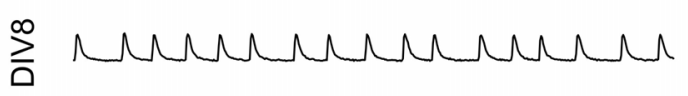
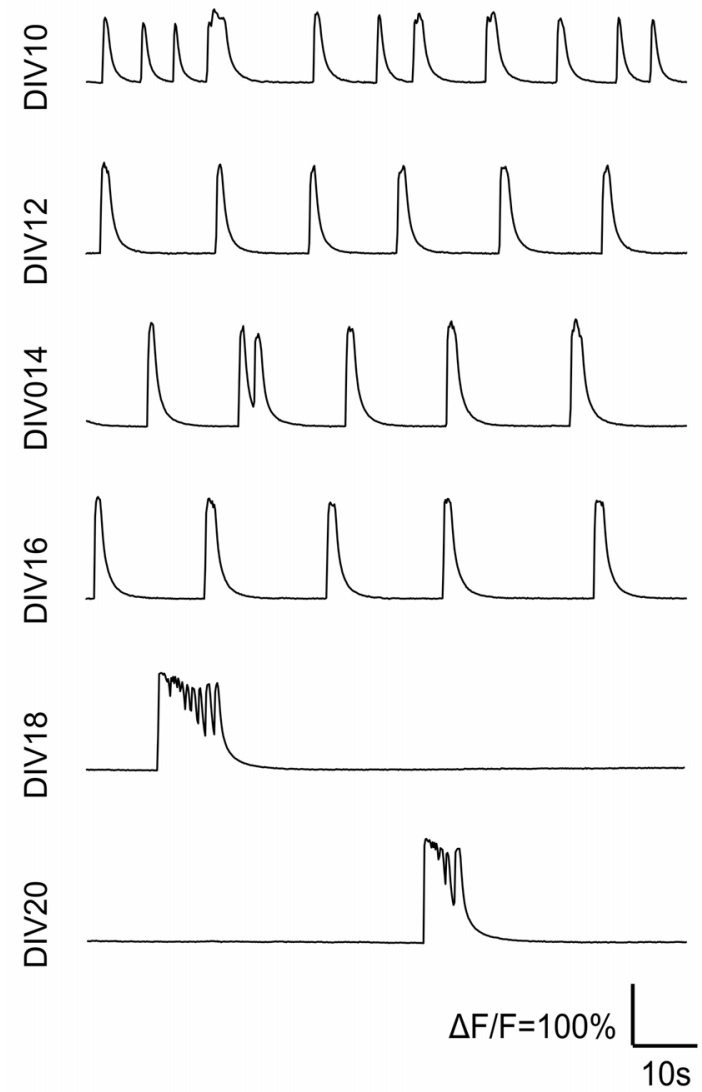

B

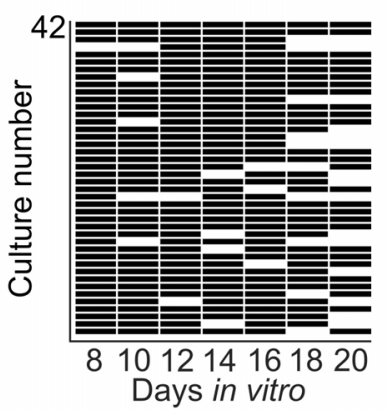

C
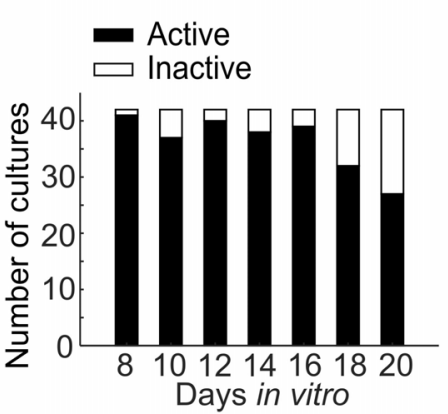

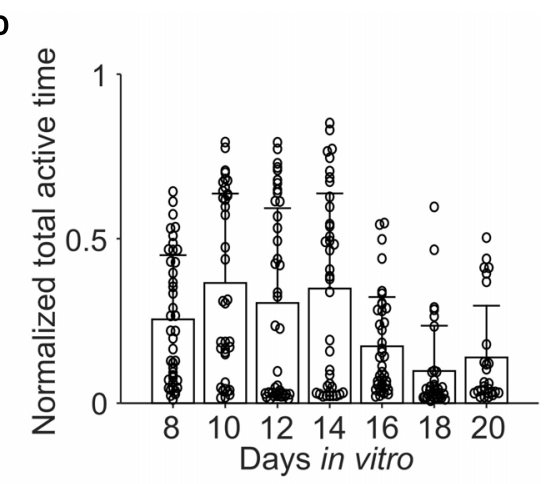

E

$\mathbf{F}$ Days in vitro
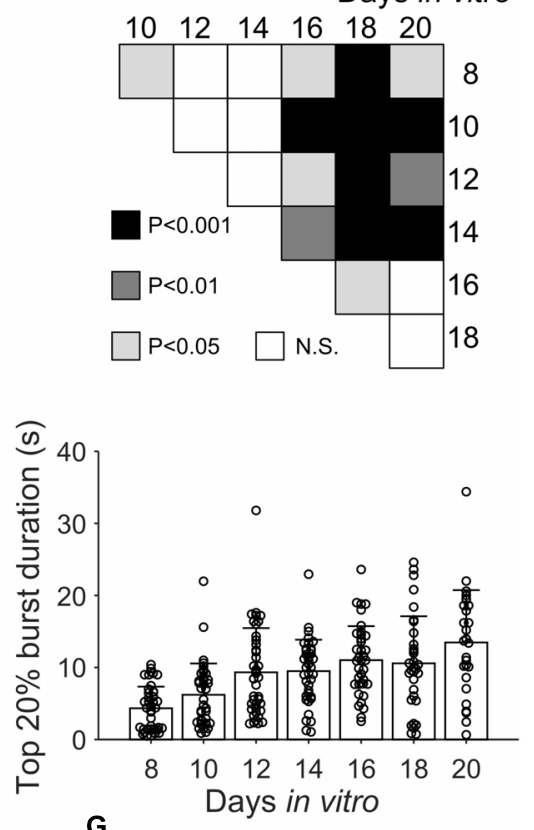

G

Days in vitro

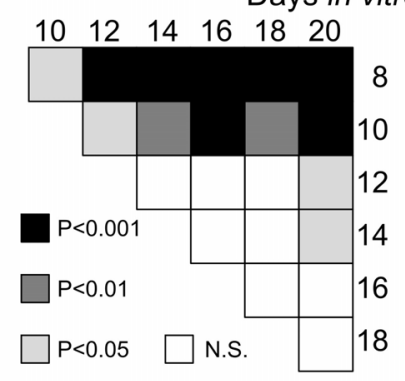

FIGURE 6 | (A) Representative $\mathrm{Ca}^{2+}$ activity of the same $\mu 3 \mathrm{D}$ culture from DIV 8 to DIV 20. (B) Map of active and inactive (during 12 min-long recording) $\mu 3 \mathrm{D}$ cultures (total culture $n=42$ from 4 batches) on different DIVs. (C) Total active and inactive cultures on different DIVs. Normalized total active time (D) and top 20\% burst duration (F) of $\mu 3 \mathrm{D}$ cultures on different DIVs. Students' t-tests of normalized total active time (E) and top $20 \%$ burst duration (G) between DIVs indicated on $\mathrm{x}$ and y axis, N.S. represents no significance. In panels (D,F), bars represent the average and error bars are standard deviations. Each circle represents a culture. $n=42$ minus number of inactive cultures for each DIV. 
processes were present throughout the $\mu 3 \mathrm{~d}$ culture volume (Figure 4C) and the ratio of astrocytes to total cells increased from $0.15 \pm 0.04$ on DIV 3 to $0.30 \pm 0.07$ on DIV18 (Figure 4E), indicating proliferation of astrocytes through time.

IV curves obtained with whole cell recordings of neurons in $\mu 3 \mathrm{D}$ cultures in current clamp mode showed that the first action potential evoked with injected current always triggered a network burst (Figure 5A, bursts triggered with 1st evoked action potential in $n=8 / 8 \mathrm{IV}$ curves from 4 neurons in 4 different cultures). When recorded in the presence of $3 \mathrm{mM}$ kynurenic acid (KYNA), there were no network bursts (Figure 5B), and IV curves were typical of cortical neurons in vitro (Barral and Reyes, 2016). Input resistance $R_{\mathrm{N}}=182.6 \pm 66.9 \mathrm{M} \Omega$, resting membrane potential $V_{\mathrm{m}}=-66.1 \pm 7.6 \mathrm{mV}, \tau_{\mathrm{m}}=23 \pm 14 \mathrm{~ms}$, threshold voltage $V_{\mathrm{t}}=-41.2 \pm 5.9 \mathrm{mV}$, threshold current $I_{\mathrm{t}}=130.6 \pm 52.7 \mathrm{pA}$, action potential (AP) half-width was $1.67 \pm 0.78 \mathrm{~ms}$, AP amplitude was $62.0 \pm 7.4 \mathrm{mV}$, afterhyperpolarization voltage was $12.33 \pm 4.0 \mathrm{mV}$, and $\mathrm{AP}$ frequency at $400 \mathrm{pA}$ injected current was $24 \pm 7.1 \mathrm{~Hz}$, average \pm standard deviation values were obtained from $n=9$ neurons in 8 cultures in the presence of $3 \mathrm{mM} \mathrm{KYNA.}$

Fluorescence changes due to activity of neurons were observed starting from about DIV 8. As cultures matured, synchronized population bursts were recorded. Dynamic fluorescence changes were recorded every 2 days from DIV 8 to DIV 20. Representative optical recordings are showed in Figure 6A. Cultures became less active on DIV 16-20 and sometimes remained silent during 12 min of optical recording (Figures 6B-E). Burst durations were measured using the method described in data analysis section. Sum of the burst durations in each active recording yielded total active time, which was then divided by the whole recording time for normalization (Figure 6D). Total active times of cultures on DIV 16-20 were significantly shorter (Student's t-test, $n=27-41$ of active cultures on corresponding DIV, Figure 6B) than earlier DIVs (Figure 6E). We then examined durations of the longest 20\% of bursts (Figure 6F). Top 20\% burst durations were significantly shorter on DIV 8-10 than DIV 12-20 and they were significantly longer on DIV 20 than DIV 8-14 (Figure 6G, Student's $t$-test, $n=27-41$ of active cultures on corresponding DIV, Figure 6B). Waveforms of bursts in $\mu 3 \mathrm{D}$ cultures were qualitatively similar (long duration, multi-peak) to bursts in large 3D cultures, but not to shorter, single peak bursts in 2D cultures (Figure 7A). Durations of the longest 20\% of bursts were significantly longer in $\mu 3 \mathrm{D}$ and large $3 \mathrm{D}$ cultures compared to $2 \mathrm{D}$ cultures (Figure 7B).

Next, we examined effects of bicuculline (on DIV21), kynurenic acid (KYNA, on DIV 19), APV and NBQX (on DIV 19), and phenytoin (on DIV 18) on the activities of $\mu 3 D$ cultures. Application of bicuculline, an antagonist of $\mathrm{GABA}_{\mathrm{A}}$ receptors (Ueno et al., 1997), decreased the number of both short and long bursts. As shown in Figures 8A,B, after application of bicuculline, durations of bursts in all microwells exhibited more compact clusters centered at middle burst duration (approximately 2-4 s). Cumulative distribution functions (CDFs) of burst duration of vehicle and bicuculline were significantly different (KS test, $p<0.001$, Figure 8C). The cross of the two CDFs at burst duration of $\sim 4 \mathrm{~s}$ indicates that there were fewer short and long

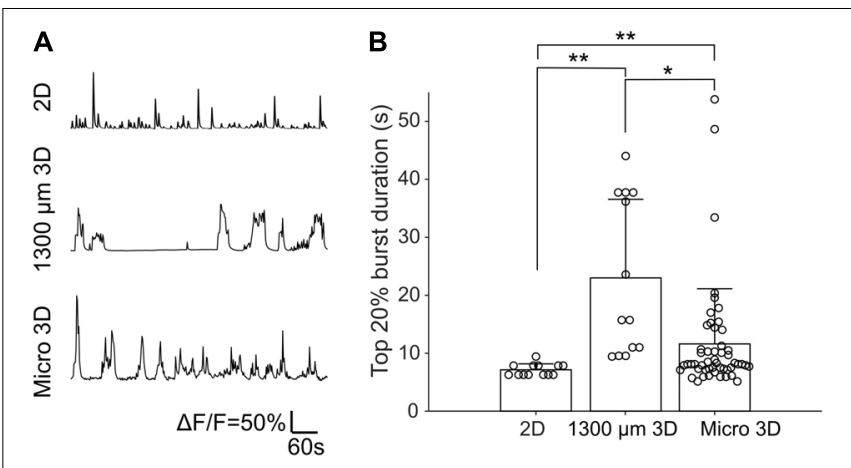

FIGURE 7 | (A) Representative $\mathrm{Ca}^{2+}$ traces of 2D, $1300 \mu \mathrm{m} 3 \mathrm{D}$ and $\mu 3 \mathrm{D}$ cultures on DIV 14. (B) Top 20\% burst durations of 2D, $1300 \mu \mathrm{m} 3 \mathrm{D}$ and $\mu 3 \mathrm{D}$ cultures on DIV 14. Data for $1300 \mu \mathrm{m}$ 3D culture has been previously published (Hasan et al., 2019) and is reproduced here for comparison. Data for $2 \mathrm{D}$ and $\mu 3 \mathrm{D}$ cultures are from experiments described in section "Materials and Methods." Bars represent the average and error bars are standard deviations. Each circle represents one detected burst. Student's $t$-tests were used to assess statistical significance. ${ }^{*} p<0.05$ and ${ }^{* *} p<0.01$. Bursts were measured from recordings of 4 cultures for each culture type.

bursts with the presence of bicuculline. On the other hand, number of bursts and normalized power of activity did not change significantly in bicuculline (Figures 8D,E). We found that KYNA, an antagonist of glutamate receptors (Moroni et al., 1988), significantly inhibited activities of the cultures (Figures 8F,G), indicating that spontaneous population bursts in $\mu 3 \mathrm{D}$ cultures are dependent on glutamatergic synapses. Presence of APV, an antagonist of NMDA receptors (Morris, 1989), significantly (Figure 8I, paired $t$-test, $p<0.001$ ) shortened the average top $20 \%$ burst durations without affecting total active time significantly (Figure $\mathbf{8 H}$ ). Blocking NMDA and AMPA receptors simultaneously with APV and NBQX completely shut down the activities. Curve fittings (Figures 8J,K) show sigmoid relationships of concentration of phenytoin, which is an antiepileptic drug that acts on voltage-gated sodium channels, to average burst durations and total active time. The activity was completely shut down at $150 \mu \mathrm{M}$ of phenytoin, and the half effective concentration $\left(\mathrm{EC}_{50}\right)$ of phenytoin is $50 \mu \mathrm{M}$.

\section{DISCUSSION}

Spontaneous activity recorded in $\mu 3 \mathrm{D}$ cultures consisted of bursts interspersed with quiet periods. Bursts lasted several seconds and contained multiple peaks. Inhibition of bursts by phenytoin indicates that the $\mathrm{Ca}^{2+}$ elevations were caused by action potentials, and that $\mathrm{Ca}^{2+}$ waveforms can be interpreted as convolution of the population neuronal firing rate with the impulse response of the $\mathrm{Ca}^{2+}$ indicator, jRGECO1a (Yaksi and Friedrich, 2006; Hasan et al., 2019). This relationship between dynamic $\mathrm{Ca}^{2+}$ in $\mu 3 \mathrm{D}$ cultures and population firing rate thus allows us to compare the spontaneous activity in this model to electroencephalogram (EEG) recordings. Qualitatively similar EEG patterns include multi-second bursts of activity separated by quiet inter-burst intervals lasting several seconds, that have 


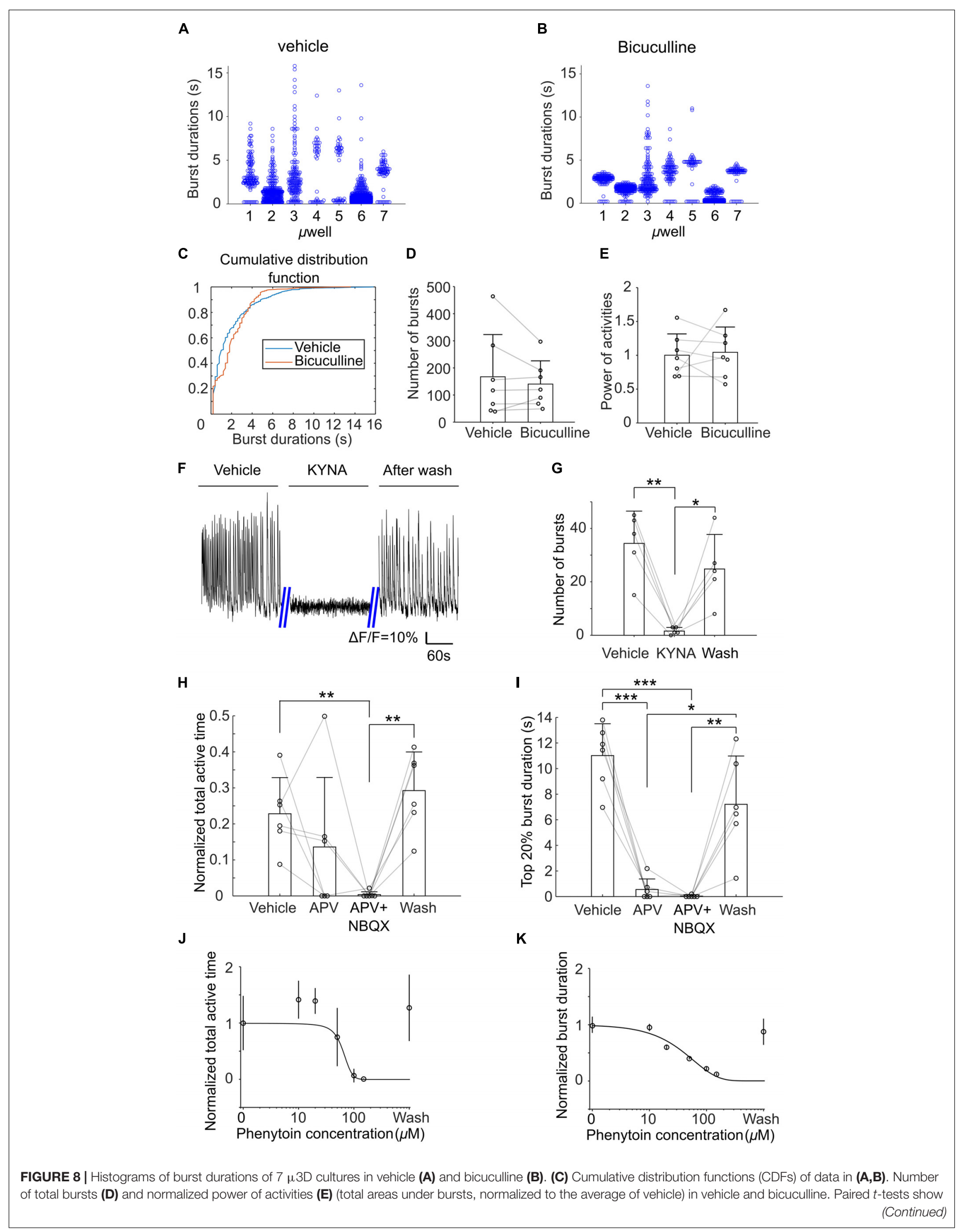




\section{FIGURE 8 | Continued}

no significance for either comparison. $n=7$ cultures, $p=0.38$ and 0.81 for panel (D,E), respectively. (F) Representative recordings of the $\mu 3 D$ culture before, during and after presence of 3 mM KYNA. (G) Number of bursts detected before, during and after KYNA application. Paired t tests were used to measure statistical significance. ${ }^{*} p<0.05$ and ${ }^{* *} p<0.01 . n=5$ cultures for each condition. Normalized total active time (H) and top $20 \%$ burst duration (I) of $\mu 3 D$ cultures during vehicle, after applying APV, after applying APV + NBQX and after wash. Paired $t$-tests were used to measure statistical significance. ${ }^{\star} p<0.05$, ${ }^{* *} p<0.01$, and ${ }^{\star \star *} p<0.001 . \mathbf{n}=6$ cultures for each condition. Normalized total active time (J) and burst duration (K) at different concentrations of phenytoin. In panels (D,E,G-I), bars represent the average and error bars are standard deviations. Each circle represents one data point. In panels (J,K), each circle represents the average of 5 cultures and error bars represent $99 \%$ confidence interval. Solid lines are curves fitted to sigmoid function y $=\mathrm{G} /\left(1+10^{\mathrm{a} 1 *(\mathrm{con}-\mathrm{a} 2)}\right)$ using least absolute residuals (LAR) method, where $\mathrm{G}=1+10^{-\mathrm{a} 1 * \mathrm{a} 2}$, con denotes the concentration of phenytoin. a1 $=3.24 \times 10^{-2}, \mathrm{a} 2=64.34, r^{2}=0.92, p=2.6 \times 10^{-3}$ for $(\mathbf{J})$ and a1 $=7.40 \times 10^{-3}$, a2 $=-1059, r^{2}=0.96, p=6.08 \times 10^{-4}$ for (K). DIVs of pharmacological experiments: bicuculline on DIV21, kynurenic acid (KYNA) on DIV 19 , APV and NBQX on DIV 19 and phenytoin on DIV 18.

been recorded from adult patients (Kroeger and Amzica, 2007; Liley and Walsh, 2013), and from otherwise healthy premature infants (André et al., 2010; Arichi et al., 2017).

In the rat cortex, oscillatory intermittent $\mathrm{Ca}^{2+}$ activity, is usually recorded at postnatal days $0-3$ (Table 1). These bursts of synchronized activity are termed cortical early network oscillations (cENOs). By postnatal day 8, cENO activity (dependent on glutamatergic synapses) disappears, and cortical giant depolarizing potentials (cGDPs), which are dependent on excitatory GABA synapses, become the dominant synchronized feature (Allene et al., 2008). In our experiments, KYNA (inhibitor of glutamate receptors) reliably blocked spontaneous population activity in $\mu 3 \mathrm{D}$ cultures on DIV 19 (Figures 8F,G). Inhibition of both NMDA and AMPA receptors was required to block spontaneous bursts. On the other hand, bicuculline (inhibitor of $\mathrm{GABA}_{\mathrm{A}}$ receptors) had significant and complex effects on burst waveform, but did not reduce the number of bursts or activity power (Figures 8A-E). This leads us to conclude that spontaneous population bursts in $\mu 3 \mathrm{D}$ cultures have the pharmacological profile of cENOs, and not cGDPs, and that developmental stage of neurons in $\mu 3 \mathrm{D}$ cultures during period of highest population activity is comparable to rat cortex during the first postnatal week.

Each $\mu 3 \mathrm{D}$ culture contains approximately 30 neurons, including around 6 GABAergic neurons on average. Our results show that this number of neurons is sufficient to produce cENOlike intermittent, long burst activity. Interestingly, number of neurons in $\mu 3 \mathrm{D}$ cultures falls within size range ( $5-50$ neurons) of synchronously active domains in the developing rat cortex (Yuste et al., 1992). Transient, prolonged burst-like elevations in $\mathrm{Ca}^{2+}$ were found in small domains of coactive neurons located within $\sim 100-200 \mu \mathrm{m}$ of each other (Golshani et al., 2009). The size of $\mu 3 \mathrm{D}$ cultures is therefore representative of the scale of domains in the developing cortex that generate spontaneous synchronized activity. Unlike in vivo cortical domains, networks in $\mu 3 \mathrm{D}$ cultures are isolated from the rest of the brain. Thus, each neuron in $\mu 3 \mathrm{D}$ culture has only about 30 potential synaptic targets. This likely leads to synaptic scaling: an increase in the strength of synaptic connection between any pair of neurons, which was previously established in $2 \mathrm{D}$ in vitro cortical networks of varying density (Barral and Reyes, 2016). In $\mu 3 \mathrm{D}$ cultures, increased synaptic strength may be the reason that network bursts were triggered by an action potential in a single neuron (Figure 5). This interesting property may make $\mu 3 \mathrm{D}$ cultures a useful model for studying mechanisms of burst initiation.
Our results show that $2 \mathrm{D}$ cultures have significantly shorter bursts than $\mu 3 \mathrm{D}$ cultures. Duration of bursts is directly related to the duration of $\mathrm{Ca}^{2+}$ elevations that neurons experience, and may thus significantly impact intracellular signaling where $\mathrm{Ca}^{2+}$ plays an important role. Large $3 \mathrm{D}$ cultures and $\mu 3 \mathrm{D}$ cultures both had significantly longer bursts than $2 \mathrm{D}$ cultures, but duration of bursts between two types of 3D cultures was similar (Figure 7). This suggests that it is the spatial arrangement and density of cells, rather than the number of cells in culture (our 2D cultures contained thousands of neurons) are important for generation of prolonged synchronized activity. Dissociated cortex contains astrocytes in addition to neurons; high packing density in $3 \mathrm{D}$ cultures may result in closer positioning of astrocytes and neurons compared to $2 \mathrm{D}$. This may influence glutamate cycling and improve neurotransmitter replenishment at the synapses, preventing burst termination due to neurotransmitter depletion (Staley et al., 1998). Other astrocyte-neuron interactions may also be involved. Higher neuronal density may result in high extracellular $\mathrm{K}^{+}$during bursts, which may also play a role in burst prolongation (Chizhov et al., 2018). Neurons and astrocytes may also provide higher trophic support for each other in 3D environment, potentially leading to higher expression of ion channels and pumps, which in turn may also contribute to burst prolongation.

In addition to modeling the size of developing cortical domains and prolonged, intermittent synchronized bursting, $\mu 3 \mathrm{D}$ culture method has a practical experimental significance. Pharmacological experiments require continuous, high framerate observation of $\mathrm{Ca}^{2+}$ for sufficient amount of time to quantify spontaneous bursts before and after drug application. This precludes the use of commercially available high-throughput systems consisting of multiple well plates and a scanning imaging system that observes one culture well at a time. Our approach was to pack many independent cultures into a field of view of an objective with sufficient power to observe fluorescence of the $\mathrm{Ca}^{2+}$ indicator. The field of view of a 10x objective is approximately $1 \times 1 \mathrm{~mm}^{2}$, close to the size of a single well of a commercially available 1536 well plate. To observe multiple cultures in a single field of view, we turned to microfabrication technology to create arrays of $\mu 3 \mathrm{D}$ cultures (Figure 2). High density arrays (100 $\mu \mathrm{m}$ culture-to-culture spacing) resulted in axon bridging between individual channels. We thus made lower density arrays with $270 \mu \mathrm{m}$ spacing to ensure that each $\mu 3 \mathrm{D}$ culture is an independent and isolated network. These arrays enabled us to obtain statistically powered results in a single 
experiment. We determined concentration response of phenytoin using $270 \mu \mathrm{m}$ arrays as a proof of principle (Figures $\mathbf{8 H}, \mathbf{I}$ ).

\section{CONCLUSION}

A method of generating $\mu 3 \mathrm{D}$ cultures in vitro is reported in this work. It was found that $\mu 3 \mathrm{D}$ cultures have more complex activity patterns than $2 \mathrm{D}$ cultures. $\mu 3 \mathrm{D}$ cultures have prolonged, intermittent population bursts with multiple peaks that are similar to human EEG patterns during development or in abnormal brain states. We also demonstrated that spontaneous activities of $\mu 3 \mathrm{D}$ cultures were dependent on glutamatergic synapses, similar to cENOs in neonatal rats. The advantages of this method are that: (1) it robustly generates a large number of $\mu 3 \mathrm{D}$ cultures in parallel, and (2) microwells can be densely packed so that drug responses of multiple cultures can be monitored simultaneously. This method has the potential to serve as an in vitro platform for screening drugs and other compounds against a phenotype of early developmental neuronal activity.

\section{DATA AVAILABILITY STATEMENT}

The raw data supporting the conclusions of this article will be made available by the authors, without undue reservation.

\section{REFERENCES}

Adelsberger, H., Garaschuk, O., and Konnerth, A. (2005). Cortical calcium waves in resting newborn mice. Nat. Neurosci. 8, 988-990. doi: 10.1038/nn1502

Allene, C., Cattani, A., Ackman, J. B., Bonifazi, P., Aniksztejn, L., Ben-Ari, Y., et al. (2008). Sequential generation of two distinct synapse-driven network patterns in developing neocortex. J. Neurosci. 28, 12851-12863. doi: 10.1523/jneurosci. 3733-08.2008

André, M., Lamblin, M.-D., d'Allest, A.-M., Curzi-Dascalova, L., MoussalliSalefranque, F., Tich, S. N. T., et al. (2010). Electroencephalography in premature and full-term infants. Developmental features and glossary. Neurophysiol. Clin. 40, 59-124. doi: 10.1016/j.neucli.2010.02.002

Arichi, T., Whitehead, K., Barone, G., Pressler, R., Padormo, F., Edwards, A. D., et al. (2017). Localization of spontaneous bursting neuronal activity in the preterm human brain with simultaneous EEG-fMRI. eLife 6:e27814.

Barral, J., and Reyes, A. D. (2016). Synaptic scaling rule preserves excitatoryinhibitory balance and salient neuronal network dynamics. Nat. Neurosci. 19, 1690-1696. doi: $10.1038 / \mathrm{nn} .4415$

Brewer, G. J., Torricelli, J., Evege, E., and Price, P. (1993). Optimized survival of hippocampal neurons in B27-supplemented neurobasal ${ }^{\mathrm{TM}}$, a new serumfree medium combination. J. Neurosci. Res. 35, 567-576. doi: 10.1002/jnr. 490350513

Casiano, C. A., Ochs, R. L., and Tan, E. M. (1998). Distinct cleavage products of nuclear proteins in apoptosis and necrosis revealed by autoantibody probes. Cell Death Differ. 5, 183-190. doi: 10.1038/sj.cdd.4400336

Chizhov, A. V., Zefirov, A. V., Amakhin, D. V., Smirnova, E. Y., and Zaitsev, A. V. (2018). Minimal model of interictal and ictal discharges "Epileptor-2". PLoS Comput. Biol. 14:e1006186. doi: 10.1371/journal.pcbi.1006186

Choi, Y. J., Park, J., and Lee, S.-H. (2013). Size-controllable networked neurospheres as a $3 \mathrm{D}$ neuronal tissue model for Alzheimer's disease studies. Biomaterials 34, 2938-2946. doi: 10.1016/j.biomaterials.2013.01.038

Colonnese, M., and Khazipov, R. (2012). Spontaneous activity in developing sensory circuits: implications for resting state fMRI. Neuroimage 62, 2212-2221. doi: 10.1016/j.neuroimage.2012.02.046

\section{ETHICS STATEMENT}

All animal use protocols were approved by the Institution Animal Care and Use Committee (IACUC) at Lehigh University and were conducted in accordance with the United States Public Health Service Policy on Humane Care and Use of Laboratory Animals.

\section{AUTHOR CONTRIBUTIONS}

YM performed the microfabrication, micro three-dimensional cell cultures, data collection and analysis and co-wrote the manuscript. MH performed the two-dimensional and large threedimensional cell cultures. ST-L participated in the design of and guided microfabrication, and revised the manuscript. YB guided the experiment design, data collection and analysis, and co-wrote the manuscript. All the authors contributed to the article and approved the submitted version.

\section{FUNDING}

This work was supported in part by the PA CURE Health Research Formula Grant 4100068719 and the Lehigh University Accelerator Grant (2014).

Conhaim, J., Easton, C. R., Becker, M. I., Barahimi, M., Cedarbaum, E. R., Moore, J. G., et al. (2011). Developmental changes in propagation patterns and transmitter dependence of waves of spontaneous activity in the mouse cerebral cortex. J. Physiol. 589, 2529-2541. doi: 10.1113/jphysiol.2010.202382

Cooper, D. J., Zunino, G., Bixby, J. L., and Lemmon, V. P. (2017). Phenotypic screening with primary neurons to identify drug targets for regeneration and degeneration. Mol. Cell. Neurosci. 80, 161-169. doi: 10.1016/j.mcn.2016.07.001

Cordey, M., Limacher, M., Kobel, S., Taylor, V., and Lutolf, M. P. (2008). Enhancing the reliability and throughput of neurosphere culture on hydrogel microwell arrays. Stem Cells 26, 2586-2594. doi: 10.1634/stemcells.2008-0498

Corlew, R., Bosma, M. M., and Moody, W. J. (2004). Spontaneous, synchronous electrical activity in neonatal mouse cortical neurones. J. Physiol. 560, 377-390. doi: 10.1113/jphysiol.2004.071621

Daud, M. F., Pawar, K. C., Claeyssens, F., Ryan, A. J., and Haycock, J. W. (2012). An aligned 3D neuronal-glial co-culture model for peripheral nerve studies. Biomaterials 33, 5901-5913. doi: 10.1016/j.biomaterials.2012. 05.008

Egorov, A. V., and Draguhn, A. (2013). Development of coherent neuronal activity patterns in mammalian cortical networks: common principles and local hetereogeneity. Mech. Dev. 130, 412-423. doi: 10.1016/j.mod.2012.09.006

Eilers, P. H., and Boelens, H. F. (2005). Baseline correction with asymmetric least squares smoothing. Leiden Univer. Med. Centre Rep. 1:5.

Frega, M., Tedesco, M., Massobrio, P., Pesce, M., and Martinoia, S. (2014). Network dynamics of 3D engineered neuronal cultures: a new experimental model for in-vitro electrophysiology. Sci. Rep. 4:5489.

Garaschuk, O., Linn, J., Eilers, J., and Konnerth, A. (2000). Large-scale oscillatory calcium waves in the immature cortex. Nat. Neurosci. 3, 452-459. doi: 10.1038/ 74823

Golshani, P., Gonçalves, J. T., Khoshkhoo, S., Mostany, R., Smirnakis, S., and Portera-Cailliau, C. (2009). Internally mediated developmental desynchronization of neocortical network activity. J. Neurosci. 29, 1089010899. doi: 10.1523/jneurosci.2012-09.2009

Hasan, M., and Berdichevsky, Y. (2016). Neural circuits on a chip. Micromachines 7:157. doi: $10.3390 / \mathrm{mi} 7090157$ 
Hasan, M. F., Ghiasvand, S., Wang, H., Miwa, J. M., and Berdichevsky, Y. (2019). Neural layer self-assembly in geometrically confined rat and human 3D cultures. Biofabrication 11:045011. doi: 10.1088/1758-5090/ab2d3f

Huang, Z., Sun, Y., Liu, W., Zhang, W., Zheng, W., and Jiang, X. (2014). Assembly of functional Three-dimensional neuronal networks on a microchip. Small 10, 2530-2536. doi: 10.1002/smll.201400513

Ichihara, Y., Utoh, R., Yamada, M., Shimizu, T., and Uchigata, Y. (2016). Size effect of engineered islets prepared using microfabricated wells on islet cell function and arrangement. Heliyon 2:e00129. doi: 10.1016/j.heliyon.2016.e00129

Jeong, G. S., Jun, Y., Song, J. H., Shin, S. H., and Lee, S.-H. (2012). Meniscus induced self organization of multiple deep concave wells in a microchannel for embryoid bodies generation. Lab Chip 12, 159-166. doi: 10.1039/c1lc20619b

Karp, J. M., Yeh, J., Eng, G., Fukuda, J., Blumling, J., Suh, K.-Y., et al. (2007). Controlling size, shape and homogeneity of embryoid bodies using poly (ethylene glycol) microwells. Lab Chip 7, 786-794. doi: 10.1039/b705085m

Kato-Negishi, M., Tsuda, Y., Onoe, H., and Takeuchi, S. (2010). A neurospheroid network-stamping method for neural transplantation to the brain. Biomaterials 31, 8939-8945. doi: 10.1016/j.biomaterials.2010.08.008

Khazipov, R., and Luhmann, H. J. (2006). Early patterns of electrical activity in the developing cerebral cortex of humans and rodents. Trends Neurosci. 29, 414-418. doi: 10.1016/j.tins.2006.05.007

Kirischuk, S., Sinning, A., Blanquie, O., Yang, J.-W., Luhmann, H. J., and Kilb, W. (2017). Modulation of neocortical development by early neuronal activity: physiology and pathophysiology. Front. Cell. Neurosci. 11:379. doi: 10.3389/ fncel.2017.00379

Kroeger, D., and Amzica, F. (2007). Hypersensitivity of the anesthesia-induced comatose brain. J. Neurosci. 27, 10597-10607. doi: 10.1523/jneurosci.3440-07. 2007

Liley, D., and Walsh, M. (2013). The mesoscopic modeling of burst suppression during anesthesia. Front. Comput. Neurosci. 7:46. doi: 10.3389/fncel.2017. 00046

Lischalk, J. W., Easton, C. R., and Moody, W. J. (2009). Bilaterally propagating waves of spontaneous activity arising from discrete pacemakers in the neonatal mouse cerebral cortex. Dev. Neurobiol. 69, 407-414. doi: 10.1002/dneu.20708

Luhmann, H. J., Sinning, A., Yang, J.-W., Reyes-Puerta, V., Stüttgen, M. C., Kirischuk, S., et al. (2016). Spontaneous neuronal activity in developing neocortical networks: from single cells to large-scale interactions. Front. Neural Circ. 10:40. doi: 10.3389/fncel.2017.00040

Marom, A., Shor, E., Levenberg, S., and Shoham, S. (2017). Spontaneous activity characteristics of 3D “optonets". Front. Neurosci. 10:602. doi: 10.3389/fncel. 2017.00602

Moeller, H.-C., Mian, M. K., Shrivastava, S., Chung, B. G., and Khademhosseini, A. (2008). A microwell array system for stem cell culture. Biomaterials 29, 752-763. doi: 10.1016/j.biomaterials.2007.10.030

Mohr, J. C., de Pablo, J. J., and Palecek, S. P. (2006). 3-D microwell culture of human embryonic stem cells. Biomaterials 27, 6032-6042. doi: 10.1016/j.biomaterials. 2006.07.012

Moroni, F., Russi, P., Lombardi, G., Beni, M., and Carla, V. (1988). Presence of kynurenic acid in the mammalian brain. J. Neurochem. 51, 177-180. doi: 10.1111/j.1471-4159.1988.tb04852.x

Morris, R. (1989). Synaptic plasticity and learning: selective impairment of learning rats and blockade of long-term potentiation in vivo by the N-methyl-Daspartate receptor antagonist AP5. J. Neurosci. 9, 3040-3057. doi: 10.1523/ jneurosci.09-09-03040.1989

Namiki, S., Norimoto, H., Kobayashi, C., Nakatani, K., Matsuki, N., and Ikegaya, Y. (2013). Layer III neurons control synchronized waves in the immature cerebral cortex. J. Neurosci. 33, 987-1001. doi: 10.1523/jneurosci.2522-12.2013

Ong, L. J. Y., Islam, A., DasGupta, R., Iyer, N. G., Leo, H. L., and Toh, Y.-C. (2017). A 3D printed microfluidic perfusion device for multicellular spheroid cultures. Biofabrication 9:045005. doi: 10.1088/1758-5090/aa8858

Park, J., Cho, C. H., Parashurama, N., Li, Y., Berthiaume, F., Toner, M., et al. (2007). Microfabrication-based modulation of embryonic stem cell differentiation. $L a b$ Chip 7, 1018-1028.

Park, J., Lee, B. K., Jeong, G. S., Hyun, J. K., Lee, C. J., and Lee, S.-H. (2015). Three-dimensional brain-on-a-chip with an interstitial level of flow and its application as an in vitro model of Alzheimer's disease. Lab Chip 15, 141-150. doi: $10.1039 / \mathrm{c} 4 \mathrm{lc} 00962 \mathrm{~b}$

Parsons, C. G. (2019). CNS repurposing-Potential new uses for old drugs: examples of screens for Alzheimer's disease, Parkinson's disease and spasticity. Neuropharmacology 147, 4-10. doi: 10.1016/j.neuropharm.2018.08.027

Pasturel, A., Strale, P.-O., and Studer, V. (2019). Tailoring 3D cell culture templates with common hydrogels. bioRxiv [Preprint], doi: 10.1101/370882

Puschmann, T. B., de Pablo, Y., Zandén, C., Liu, J., and Pekny, M. (2014). A novel method for three-dimensional culture of central nervous system neurons. Tissue Eng. Part C Methods 20, 485-492. doi: 10.1089/ten.tec.2013.0445

Sakai, Y., Yoshida, S., Yoshiura, Y., Mori, R., Tamura, T., Yahiro, K., et al. (2010). Effect of microwell chip structure on cell microsphere production of various animal cells. J. Biosci. Bioeng. 110, 223-229. doi: 10.1016/j.jbiosc.2010. 01.021

Schneider, C. A., Rasband, W. S., and Eliceiri, K. W. (2012). NIH Image to ImageJ: 25 years of image analysis. Nat. Methods 9:671. doi: 10.1038/nmeth.2089

Selimović, Š, Piraino, F., Bae, H., Rasponi, M., Redaelli, A., and Khademhosseini, A. (2011). Microfabricated polyester conical microwells for cell culture applications. Lab Chip 11, 2325-2332. doi: 10.1039/c1lc20213h

Sheroziya, M. G., Halbach, O. V. B., Unsicker, K., and Egorov, A. V. (2009). Spontaneous bursting activity in the developing entorhinal cortex. J. Neurosci. 29, 12131-12144. doi: 10.1523/jneurosci.133309.2009

Staley, K. J., Longacher, M., Bains, J. S., and Yee, A. (1998). Presynaptic modulation of CA3 network activity. Nat. Neurosci. 1, 201-209. doi: 10.1038/651

Tung, Y.-C., Hsiao, A. Y., Allen, S. G., Torisawa, Y.-S., Ho, M., and Takayama, S. (2011). High-throughput 3D spheroid culture and drug testing using a 384 hanging drop array. Analyst 136, 473-478. doi: 10.1039/c0an00609b

Ueno, S., Bracamontes, J., Zorumski, C., Weiss, D. S., and Steinbach, J. H. (1997). Bicuculline and gabazine are allosteric inhibitors of channel opening of the GABAA receptor. J. Neurosci. 17, 625-634. doi: 10.1523/jneurosci.17-0200625.1997

van Pelt, J., Wolters, P. S., Corner, M. A., Rutten, W. L., and Ramakers, G. J. (2004). Long-term characterization of firing dynamics of spontaneous bursts in cultured neural networks. IEEE Trans. Biomed. Eng. 51, 2051-2062. doi: 10.1109/tbme.2004.827936

Vanhatalo, S., Palva, J. M., Andersson, S., Rivera, C., Voipio, J., and Kaila, K. (2005). Slow endogenous activity transients and developmental expression of $\mathrm{K}+-\mathrm{Cl}-$ cotransporter 2 in the immature human cortex. Eur. J. Neurosci. 22, 2799-2804. doi: 10.1111/j.1460-9568.2005.04459.x

Wagenaar, D. A., Pine, J., and Potter, S. M. (2006). An extremely rich repertoire of bursting patterns during the development of cortical cultures. BMC Neurosci. 7:11. doi: 10.1186/1471-2202-7-11

Wevers, N. R., Van Vught, R., Wilschut, K. J., Nicolas, A., Chiang, C., Lanz, H. L., et al. (2016). High-throughput compound evaluation on 3D networks of neurons and glia in a microfluidic platform. Sci. Rep. 6, $1-10$.

Yaksi, E., and Friedrich, R. W. (2006). Reconstruction of firing rate changes across neuronal populations by temporally deconvolved Ca $2+$ imaging. Nat. Methods 3, 377-383. doi: 10.1038/nmeth874

Yang, J.-W., Reyes-Puerta, V., Kilb, W., and Luhmann, H. J. (2016). Spindle bursts in neonatal rat cerebral cortex. Neural Plast. 2016:3467832.

Yuste, R., Peinado, A., and Katz, L. C. (1992). Neuronal domains in developing neocortex. Science 257, 665-669. doi: 10.1126/science.1496379

Conflict of Interest: The authors declare that the research was conducted in the absence of any commercial or financial relationships that could be construed as a potential conflict of interest.

Copyright (c) 2020 Ming, Hasan, Tatic-Lucic and Berdichevsky. This is an open-access article distributed under the terms of the Creative Commons Attribution License (CC BY). The use, distribution or reproduction in other forums is permitted, provided the original author(s) and the copyright owner(s) are credited and that the original publication in this journal is cited, in accordance with accepted academic practice. No use, distribution or reproduction is permitted which does not comply with these terms. 